\title{
Herbal Medicine for Cancer Treatment: Main Force or Supplement?
}

ISSN: 2637-773X

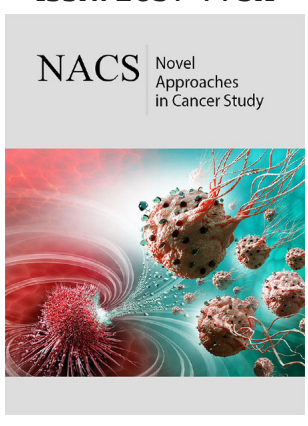

*Corresponding author: Wing Shing Ho, School of Life Sciences, Shatin, Hong Kong

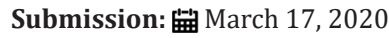

Published: 陋April 06, 2020

Volume 4 - Issue 3

How to cite this article: Wing Shing Ho. Herbal Medicine for Cancer Treatment: Main Force or Supplement?. Nov Appro in Can Study. 4(3). NACS.000587.2020. DOI: 10.31031/NACS.2020.04.000587

Copyright@ Wing Shing Ho. This article is distributed under the terms of the Creative Commons Attribution 4.0 International License, which permits unrestricted use and redistribution provided that the original author and source are credited.

\author{
Wing Shing Ho* \\ School of Life Sciences, Hong Kong
}

\section{Opinion}

The Estimated numbers of new cancer cases and deaths in 2020 will be an estimated. 1.8 million new cancer cases diagnosed and 606,520 cancer deaths in the United States and there were 17 million new cases of cancer worldwide in 2018 [1]. The four most common cancers occurring worldwide are lung, female breast, bowel and prostate. A person's risk of developing cancer depends on different factors, including age, genetics, and exposure to some potentially known carcinogens and those which are believed to be carcinogenic. Some avoidable lifestyle factors including smoking can increase cancer risk. However, cancer risk factors are similar worldwide. Insufficient exercise, alcohol, imbalanced diet, and obesity, and various infections account for a considerable proportion of cancers worldwide. Prevalence of any one of the risk factors varies in different ethnic groups and can vary by region and country, which contributes to variation of cancer incidence rates of the common types of cancer worldwide.

With no novel drugs to treat cancer are available, new approach in cancer therapy is warranted.

To help lower both the death and diagnosis rates, the American Cancer Society (ACS) and the top health organizations and other pharmaceutics fund the research of scientists across the country who use the latest evidence and cutting-edge technology to help prevent and treat cancer. There are several FDA- approved vaccines that help protect against viruses that are known to cause certain types of cancer including human papillomavirus and hepatitis B. However, creating a vaccine to prevent cancer itself has its own difficulties and it is costly. When a virus enters the body, the immune system can recognize it as a foreign invader and attack it. But the immune system has a harder time to know a cancer cell as a foreign invader because it's often not as easy to recognize the abnormal cells. Nevertheless, it is well known that the immune system can be mobilized to fight against various diseases and cancer but what approaches can be adopted to effectively strengthen the immune system with herbal medicine for the treatment methods. While significant improvements have been made in recent years to the survival rates for cancer patients, clearly we still have a long way to go for curative treatment for all cancers. Only through intensive research into novel and innovative approaches to treat cancer, can we move forwards to achieve a satisfactory treatment for all cancers.

Cancer develops when one cell suffers damage to its DNA which can undergo multiple types of damage. In most cases, our cells have the capacity to repair DNA damage. However, in some cases the damage is beyond repair. This results in mutations which ultimately leads to uncontrolled cellular growth, and hence cancer. The common approach to inhibit cancer growth is to employ integrated medicine based on western medicine and traditional Chinese medicine which has become an important step in cancer treatment of individual patients because of the advantages of using personalized medicine and minimizing the side effects of cancer drugs.

Another remarkable health benefit of using TCM is their multi-arrays of pharmacological activity in the body and with little side effects on long term use. The China's top health organization and pharmaceutics have used various treatment methods including cancer drug 
in combination with traditional Chinese medicine in cancer therapy. The National Health Commission of the People's Republic of China and the National Administration of Traditional Chinese Medicine of the People's Republic of China pioneered a diagnosis and treatment plan for novel coronavirus pneumonia on January 27, 2020, which has provided specific recommendations for the treatment and prevention of the infection using TCM. With no effective drugs to treat the infections, a novel approach in treatment of coronavirus pneumonia is highly warranted.

It is widely advocated to adopt the use of integrated Chinese and Western medicine in cancer therapy. However, cautions must be exercised when both medicines are administered simultaneously due to drug interactions. But there's no evidence of significant side effects with the combination therapy. TCM has been used against different cancers for ages. In ancient Chinese medical book Huang Di Nei Jing (Inner Canon of the Yellow Emperor), methods and drugs (Xiao Jin Pill) for preventing cancer have been described [2]. The use of TCM for treatment of cancer provides insights into treatment of other diseases and infections. TCM offers promise for treatment of various cancers worldwide.

\section{References}

1. Siegel RL, Miller KD, Jemal A (2020) Cancer Statistics

2. https://www.tmrjournals.com/tmr/EN/10.12032/TMR20200204158 